\title{
Tissue storage of dipeptides as protein guards against oxidative injury in patients with type 2 diabetes and its micro vascular complications
}

\begin{abstract}
Histidine-containing dipeptides such as Carnosine ( $\beta$-alanine-L-histidine) and anserine ( $\beta$-alanine-L-methyl histidine) are not incorporated into proteins; instead, they are stored in peripheral skeletal muscle and other organs, including the kidney, retina, and myocardium. Carnosine has several protective functions in health and disease state. This mini review provides a current overview of the knowledge of the histidine containing dipeptides in disease accompanies by oxidative stress like diabetes and diabetic micro vascular complications. It also highlights the performed experimental studies of histidine-containing dipeptides in relation to tissue damage related to (noninvasive) human studies. Finally, it describes future perspectives of the therapeutic use of histidine-containing dipeptides in health and disease.
\end{abstract}

Keywords: histidine-containing dipeptides, oxidative stress/injury, carnosine, carnosinase, diabetes, diabetic micro vascular complications
Volume I Issue 3 - 20I5

\author{
Celine Klessens QF, Hans Baelde J, Emile de \\ Heer
}

Department of Pathology, Leiden University Medical Center, Netherlands

Correspondence: Celine Klessens QF, Department of Pathology, Leiden University Medical Center, LIQ Room P0-107, P.O. Box 9600,2300 RC Leiden the Netherland, Tel 3I(0)7I-526 5008 Fax +3l (0)7l-526 6952, Email C.F.Q.Klessens@lumc.nl

Received: August 8, 2015 | Published: September 28, 2015
Abbreviations: ROS: Reactive Oxygen Species; TGF: Transforming Growth Factor; CARNS: Carnosine Synthase; CNDP1: Carnosinase-1; DN: Diabetic Nephropathy; AGE: Advanced Glycation End-Product

\section{Introduction}

Type 2 diabetes mellitus affects more than 400million patients worldwide ${ }^{1}$. The disturbed glucose metabolism in this metabolic disorder results in increased production of reactive oxygen species (ROS), and in oxidative systemic damage, accompanied by reduced defense and repair mechanisms against oxidative injury. ${ }^{2-5}$ In addition the development of micro vascular complications of diabetes in a subset of patients $(30-50 \%)^{6}$ such as diabetic nephropathy, retinopathy and cardiomyopathy have been associated with excessive oxidative stress. In several studies oxidative injury has been implicated as a driving force in micro vascular complications. ${ }^{7-9}$ Since oxidative stress, carbonyl stress, and transforming growth factor (TGF)-beta hyperactivity are prominent features in patients with type 2diabetes, new treatment modalities should be focused on natural repair mechanisms that are beneficial to diabetic patients.

Histidine-containing dipeptides such as Carnosine ( $\beta$-alanineL-histidine) and anserine ( $\beta$-alanine-L-methyl histidine) are stored in high concentrations in various tissues. These dipeptides are not incorporated into proteins; instead they are stored in peripheral skeletal muscle cells and other organs, including the kidney, pancreas, retina, and myocardium. $\beta$-alanine is the rate limiting amino acid in the biosynthesis of these histidine-containing peptides, but can be provided by oral intake. $\beta$-Alanine is internalized by cells via the taurine transporter in order to be synthesized into Carnosine or anserine for subsequent intracellular storage. ${ }^{10,11}$ Carnosine is synthesized by the enzyme Carnosine synthase (CARNS). ${ }^{12}$ The gene that encodes CARNS is ATPGD $1,{ }^{13}$ the expression and distribution of CARNS and ATPGD1 require additional studies. ${ }^{14}$ In primates, carnosine is degraded predominantly by the enzyme carnosinase- 1 (CNDP1), which is synthesized and secreted by the liver into the circulation. CNDP1 is encoded by the CNDP1 gene (Figure 1). ${ }^{15}$ In rodents, CNDP1 is absent in the circulation. Two forms of Carnosinase are expressed in primates: carnosinase-1, which is also called serum Carnosinase, and carnosinase-2, which is also called tissue Carnosinase or cytosolic nonspecific dipeptides. ${ }^{16}$

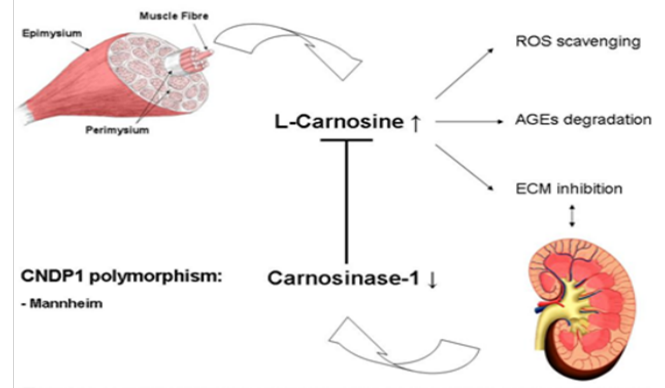

Figure I CNDPI polymorphism can lead to diminished carnosinase- Imediated cleavage of L-carnosine, thereby stimulating potential antagonistic pathways in the development of diabetic nephropathy.

The highest concentration of carnosine can be found in the skeletal muscle $(7.2-30.7 \mathrm{mmol} / \mathrm{kg}$ dry muscle weight). Carnosine are secreted into the circulation by skeletal muscle cells during physical exercise, which is in line with the beneficial effects of physical exercise on diabetic complications. ${ }^{17,18}$ Our recent study shows that the kidney has its own carnosine metabolism. ${ }^{19}$ The storage form in the human kidney is anserine $(1.1-7.4 \mathrm{mmol} / \mathrm{kg}$ for anserine). Carnosine and anserine have several protective functions such as buffering of $\mathrm{pH}$, scavenging of reactive oxygen species, ${ }^{20-22}$ degradation of advanced Glycation end-products, ${ }^{23}$ inhibition of mesangial cell proliferation, ${ }^{24}$ and inhibition of TGF-beta-mediated transcription of extracellular matrix proteins in both podocytes ${ }^{25}$ and mesangial cells..$^{26}$ All of these reported properties of carnosine can be beneficial in patients with diabetes. With respect to the genetic predisposition for complications such as diabetic nephropathy (DN), a polymorphism in exon 2 of the CNDP1 gene (homozygosity for 5-5 Leuicine repeats) is associated 
with low serum concentrations of CNDP1 and a reduced susceptibility for developing DN, ${ }^{27}$ especially in women with type 2diabetes. ${ }^{28}$

Under normal physiological conditions the production of ROS (for instance during physical exercise) are neutralised with antioxidants like carnosine and do not result in oxidative damage. ${ }^{29}$ Any imbalance between these pro-oxidants and antioxidants can result in cellular disruption and damage, and might lead to diseases like diabetes. ${ }^{30}$ In patients with excessive obesity, pre-diabetes and decreased insulin sensitivity, but without micro vascular complications the storage of carnosine in skeletal muscles has increased. ${ }^{31}$ In contrast, in patients with type 2 diabetes with micro vascular complications a significant reduction of carnosine in skeletal muscles has been reported, while this reduction remains absent in patients with type 1 diabetes. ${ }^{32}$ Multiple studies confirmed the hypertensive and antihypertensive effect of carnosine ${ }^{33,34}$ and the induction of $\mathrm{Ca}^{2+}$ release. ${ }^{35}$ Considering this vascular effect, we hypothesize that sufficient carnosine storage might result in the prevention of micro vascular complications of diabetes, related to oxidative injury.

\section{Discussion and future perspectives}

Carnosines have been used as food additives for many years in explosion sports such as sprinting, rowing, short track speed skating. Since increased carnosine storage results in reduced muscle pain and fatigue after physical exercise, ${ }^{36-39}$ many athletes are using betaalanine as a food supplement. The therapeutic effects of increased carnosine storage have been barely explored in diseases such as diabetes, which are characterized by oxidative injury. Clinical trials are required to investigate the therapeutic potential of these histidine-containing dipeptides in (diabetic) patients. The functional properties of carnosine and anserine and the genetic association with DN indicate that histidine-containing dipeptides have a protective function concerning tissue damage by oxidative stress. Therefore, we hypothesize that higher concentrations of these histidine-containing dipeptides are beneficial in attenuating oxidative injury by quenching oxygen radicals. Experimental studies in rodents already investigated the overall protective effect of carnosine, but species differences need to be taken into consideration because of the absence of CNDP1 in sera of rodents. ${ }^{40}$ Experiments in diabetic rats and mice have shown that oral administration of carnosine results in prevention of $\mathrm{DN}^{25,41,42}$ and cataract ${ }^{43,44}$ and they accelerated wound repair. ${ }^{45}$ Oral supplementation of carnosine in the drinking water of mice with DN results in accumulation of carnosine in muscular tissues and kidneys, reduction of proteinuria, and increased biosynthesis of insulin..$^{25}$

In humans, taking oral supplements of slow-release beta-alanine tablets drives to de novo biosynthesis of carnosine, leading to increased carnosine storage in tissues. ${ }^{46-50}$ Carnosines, which are secreted into the circulation by skeletal muscle cells during physical exercise, show similar beneficial effects as physical exercise on diabetic complications. ${ }^{14,51}$ Enzyme measurements for CNDP1 in human sera of healthy adults and diabetic patients have provided indications for post-translational modifications of CNDP1, which influence its prolonged enzyme activity by additional glycosylation. ${ }^{46}$ In diabetic patients with microvascular complications, non-invasive measurement of tissue carnosine concentrations by proton magnetic resonance spectroscopy (1H-MRS) shows a significant reduction of carnosine concentrations in skeletal muscles of patients with type 2diabetes, but not in patients with type 1 diabetes. ${ }^{36}$ Oral administration of $\beta$-alanine, the rate limiting amino acid for the biosynthesis of Carnosine ${ }^{48}$ to healthy volunteers during 4weeks resulted in reduced fatigue after physical exercise and in $30-50 \%$ increase of carnosine storage in peripheral muscle tissue, as determined again by proton-MRS. ${ }^{52}$ Interestingly, this accumulation is followed by a slow clearance of carnosine during 3-6months, suggesting a prolonged efficacy of short-term intervention. ${ }^{49,53}$ Next, the latter mentioned study also showed that in humans the histidine-containing dipeptides increase with increasing obesity and glucose intolerance in male individuals. They hypothesize that the skeletal muscle tissue responds differently compared to retina, kidney and liver, because of the high amount of CARNS, which can restore the decreased Carnosine levels more easily in the muscles. ${ }^{31}$ Still, more research is required to determine the beneficial role of Carnosine in humans.

\section{Conclusion}

The results of several experimental studies in rodents as well as human studies indicate the possible protective role of these histidinecontaining dipeptides. The ability of carnosine to reserve protein glycation and to inhibit AGEs formation might be the underlying mechanism of the protection in oxidative damage. Still, it is difficult to extrapolate the experimental results into humans because of the lack of CNDP1 in sera of rodents. Microvascular complications of diabetes develop in at least $30 \%$ of the diabetes patients. Our hypothesis is that carnosine could play a role in the natural resistance against diabetic damage. We postulate that in the $70 \%$ of the diabetic patients no complications occur because of the compensatory increased accumulation of carnosine. In patients with diabetic microvascular complications carnosine storage is not maintained either by insufficient activity of CARNS, or by high activity of CNDP1 in tissue, but more research is needed to confirm these hypotheses. The association between diabetic tissue damage and the improvement after oral supplementation provides evidence for future therapeutic perspectives in treatment of microvascular complications of diabetes.

\section{Acknowledgements}

None.

\section{Conflict of interest}

The author declares no conflict of interest.

\section{References}

1. International Diabetes Federation. 6th ed. IDF Diabetes Atlas; 2013.

2. Martín-Gallán P, Carrascosa A, Gussinyé M, et al. Biomarkers of diabetesassociated oxidative stress and antioxidant status in young diabetic patients with or without subclinical complications. Free Radic Biol Med. 2003;34(12):1563-1574.

3. Varvarovská J, Racek J, Stozický F, et al. Parameters of oxidative stress in children with Type 1 diabetes mellitus and their relatives. $J$ Diabetes Complications. 2003;17(1):7-10.

4. Seghrouchni I, Drai J, Bannier E, et al. Oxidative stress parameters in type I, type II and insulin-treated type 2 diabetes mellitus; insulin treatment efficiency. Clin Chim Acta. 2002;321(1-2):89-96.

5. VanderJagt DJ1, Harrison JM, Ratliff DM, et al. Oxidative stress indices in IDDM subjects with and without long-term diabetic complications. Clin Biochem. 2001;34(4):265-270.

6. Reutens AT, Atkins RC. Epidemiology of diabetic nephropathy. Contrib Nephrol. 2011;170:1-7. 
7. Rösen P1, Nawroth PP, King G, et al. The role of oxidative stress in the onset and progression of diabetes and its complications: a summary of Congress Series sponsored by UNESCO-MCBN, the American diabetes association and the german diabetes society. Diabetes Metab Res Rev. 2001;17(3):189-212.

8. Nishikawa T, Edelstein D, Brownlee M. The missing link: a single unifying mechanism for diabetic complications. Kidney Int Suppl. 2001;77:S26S30.

9. West IC. Radicals and oxidative stress in diabetes. Diabet Med. 2000;17(3):171-180.

10. Jessen H. Taurine and beta-alanine transport in an established human kidney cell line derived from the proximal tubule. Biochim Biophys Acta. 1994;1194(1):44-52.

11. Jessen H, Sheikh MI. Renal transport of taurine in luminal membrane vesicles from rabbit proximal tubule. Biochim Biophys Acta. 1991;1064(2):189-198.

12. Veiga-da-Cunha M, Chevalier N, Stroobant V, et al. Metabolite proofreading in carnosine and homocarnosine synthesis: molecular identification of PM20D2 as beta-alanyl-lysine dipeptidase. J Biol Chem. 2014;289(28):19726-1936.

13. Drozak J, Veiga-da-Cunha M, Vertommen D, et al. Molecular identification of carnosine synthase as ATP-grasp domain-containing protein 1 (ATPGD1). J Biol Chem. 2010;285(13):9346-9356.

14. Boldyrev AA, Aldini G, Derave W. Physiology and pathophysiology of carnosine. Physiol Rev. 2013;93(4):1803-1845.

15. Teufel M, Saudek V, Ledig JP, et al. Sequence identification and characterization of human carnosinase and a closely related non-specific dipeptidase. J Biol Chem. 2003;278(8):6521-6531.

16. Teufel M, Roggentin P, Schauer R. Properties of sialidase isolated from Actinomyces viscosus DSM 43798. Biol Chem Hoppe Seyler. 1989;370(5):435-443.

17. Lazarevic G1, Antic S, Vlahovic P, et al. Effects of aerobic exercise on microalbuminuria and enzymuria in type 2 diabetic patients. Ren Fail. 2007;29(2):199-205.

18. Reid RD, Tulloch HE, Sigal RJ, et al. Effects of aerobic exercise, resistance exercise or both, on patient-reported health status and well-being in type 2 diabetes mellitus: a randomised trial. Diabetologia. 2010;53(4):632-640.

19. Peters V, Klessens CQ, Baelde HJ, et al. Intrinsic carnosine metabolism in the human kidney. Amino Acids. 2015:47(12):2541-2550.

20. Barski OA, Xie Z, Baba SP, et al. Dietary carnosine prevents early atherosclerotic lesion formation in apolipoprotein E-null mice. Arterioscle Thromb Vasc Biol. 2013;33(6):1162-1170.

21. Negre-Salvayre A, Coatrieux C, Ingueneau C, et al. Advanced lipid peroxidation end products in oxidative damage to proteins. Potential role in diseases and therapeutic prospects for the inhibitors. Br J Pharmacol. 2008;153(1):6-20.

22. Vistoli G, Orioli M, Pedretti A, et al. Design, synthesis, and evaluation of carnosine derivatives as selective and efficient sequestering agents of cytotoxic reactive carbonyl species. Chem Med Chem. 2009;4(6):967-975.

23. Alhamdani MS, Kassir AH, Abbas FK, et al. Antiglycation and antioxidan effect of carnosine against glucose degradation products in peritoneal mesothelial cells. Nephron Clin Pract. 2007;107(1):c26-34.

24. Jia H, Qi X, Fang S, et al. Carnosine inhibits high glucose-induced mesangial cell proliferation through mediating cell cycle progression. Regul Pept. 2009;154(1-3):69-76.

25. Riedl E, Pfister F, Braunagel M, et al. Carnosine prevents apoptosis of glomerular cells and podocyte loss in STZ diabetic rats. Cell Physiol Biochem. 2011;28(2):279-288.

26. Köppel H, Riedl E, Braunagel M, et al. L-carnosine inhibits high-glucosemediated matrix accumulation in human mesangial cells by interfering with TGF-beta production and signalling. Nephrol Dial Transplant. 2011;26(12):3852-3858.

27. Janssen B, Hohenadel D, Brinkkoetter P, et al. Carnosine as a protective factor in diabetic nephropathy: association with a leucine repeat of the carnosinase gene CNDP1. Diabetes. 2005;54(8):2320-2327.

28. Mooyaart AL, Zutinic A, Bakker SJ, et al. Association between CNDP1 genotype and diabetic nephropathy is sex specific. Diabetes. 2010;59(6):1555-1559.

29. Turrens JF, Boveris A. Generation of superoxide anion by the NADH dehydrogenase of bovine heart mitochondria. Biochem J. 1980;191(2):421427.

30. Sies H. Oxidative stress: oxidants and antioxidants. Exp Physiol. 1997;82(2):291-295.

31. Stegen S, Everaert I, Deldicque L, et al. Muscle histidine-containing dipeptides are elevated by glucose intolerance in both rodents and men. PLoS One. 2015;10(3):e0121062.

32. Gualano B, Everaert I, Stegen S, et al. Reduced muscle carnosine content in type 2, but not in type 1 diabetic patients. Amino Acids. 2012;43(1):2124.

33. Guney Y, Turkcu UO, Hicsonmez A, et al. Carnosine may reduce lung injury caused by radiation therapy. Med Hypotheses. 2006;66(5):957-959.

34. Niijima A, Okui T, Matsumura Y, et al. Effects of L-carnosine on renal sympathetic nerve activity and DOCA-salt hypertension in rats. Auton Neurosci. 2002;97(2):99-102.

35. Gordon AM, Homsher E, Regnier M. Regulation of contraction in striated muscle. Physiol Rev. 2000;80(2):853-924.

36. Derave W, Ozdemir MS, Harris RC, et al. beta-Alanine supplementation augments muscle carnosine content and attenuates fatigue during repeated isokinetic contraction bouts in trained sprinters. J Appl Physiol (1985) 2007;103(5):1736-1743.

37. Artioli GG, Gualano B, Smith A, et al. Role of beta-alanine supplementation on muscle carnosine and exercise performance. Med Sci Sports Exerc. 2010;42(6):1162-1673.

38. Kendrick IP, Kim HJ, Harris RC, et al. The effect of 4 weeks beta-alanine supplementation and isokinetic training on carnosine concentrations in type I and II human skeletal muscle fibres. Eur J Appl Physiol. 2009;106(1):131-138.

39. del Favero S, Roschel H, Solis MY, et al. Beta-alanine (Carnosyn) supplementation in elderly subjects (60-80years): effects on muscle carnosine content and physical capacity. Amino Acids. 2012;43(1):49-56.

40. Lee YT, Hsu CC, Lin MH, et al. Histidine and carnosine delay diabetic deterioration in mice and protect human low density lipoprotein against oxidation and glycation. Eur J Pharmacol. 2005;513(1-2):145-150.

41. Aldini G1, Orioli M, Rossoni G, et al. The carbonyl scavenger carnosine ameliorates dyslipidaemia and renal function in Zucker obese rats. J Cell Mol Med. 2011;15(6):1339-1954.

42. Peters V, Schmitt CP, Zschocke J, et al. Carnosine treatment largely prevents alterations of renal carnosine metabolism in diabetic mice. Amino Acids. 2012;42(6):2411-2416

43. Yan H, Guo Y, Zhang J, et al. Effect of carnosine, aminoguanidine, and aspirin drops on the prevention of cataracts in diabetic rats. Mol Vis. $2008 ; 14: 2282-2291$ 
44. Pfister F, Riedl E, Wang Q, et al. Oral carnosine supplementation prevents vascular damage in experimental diabetic retinopathy. Cell Physiol Biochem. 2011;28(1):125-136.

45. Ansurudeen I, Sunkari VG, Grünler J, et al. Carnosine enhances diabetic wound healing in the $\mathrm{db} / \mathrm{db}$ mouse model of type 2 diabetes. Amino Acids. 2012;43(1):127-134.

46. Derave W, Everaert I, Beeckman S, et al. Muscle carnosine metabolism and beta-alanine supplementation in relation to exercise and training. Sports Med. 2010;40(3):247-263.

47. Stegen $\mathrm{S}, \mathrm{Bex} \mathrm{T}$, Vervaet $\mathrm{C}$, et al. beta-Alanine dose for maintaining moderately elevated muscle carnosine levels. Med Sci Sports Exerc. 2014;46(7):1426-1432.

48. Stellingwerff T, Anwander H, Egger A, et al. Effect of two beta-alanine dosing protocols on muscle carnosine synthesis and washout. Amino Acids. 2012;42(6):2461-2472.
49. Décombaz J, Beaumont $\mathrm{M}$, Vuichoud J, et al. Effect of slow-release beta-alanine tablets on absorption kinetics and paresthesia. Amino Acids. 2012;43(1):67-76.

50. Stellingwerff T, Decombaz J, Harris RC, et al. Optimizing human in vivo dosing and delivery of beta-alanine supplements for muscle carnosine synthesis. Amino Acids. 2012;43(1):57-65.

51. Aldini G, Facino RM, Beretta G, et al. Carnosine and related dipeptides as quenchers of reactive carbonyl species: from structural studies to therapeutic perspectives. Biofactors. 2055;24(1-4):77-87.

52. Everaert I, Taes Y, De Heer E, et al. Low plasma carnosinase activity promotes carnosinemia after carnosine ingestion in humans. Am J Physiol Renal Physiol. 2012;302(12):F1537-1544.

53. Baguet A, Reyngoudt H, Pottier A, et al. Carnosine loading and washout in human skeletal muscles. J Appl Physiol. 2009;106(3):837-842. 